\title{
Tumores Malignos del Cuerpo Uterino y Coriocarcinoma
}

\author{
Dr. Francisco S. Orozco Riquett* \\ Departamento de Obstetricia y Ginecología. - Hospital de San Juan de Dios \\ Bogotá, Enero de 1960

\section{A - MATERIAL}

Es éste un trabajo de recopilación estadística sobre quince historias clínicas de neoplasias malignas del cuerpo uterino discriminadas así: diez adenocarcinomas y cinco coriocarcinomas, encontradas en el Hospital de San Juan de Dios en un lapso comprendido entre 1953 y 1959.

\section{ADENOCARCINOMA DEL ENDOMETRIO}

\section{B - DEFINICIONES}

Se conoce como adenocarcinoma del endometrio un tumor originado en el revestimiento glándulo-epitelial que recubre la cavidad endouterina, revestimiento que activamente se modifica en forma periódica por influencias hormonales y que tiene como límites anatómicos los ostiums tubáricos y el orificio interno del cuello.

\section{$\mathrm{C}-$ FRECUENCIA}

Los libros de texto de Ginecología, dentro de grandes variaciones, reconocen una menor frecuencia del adenocarcinoma en relación con el carcinoma del cuello. El Adenocarcinoma Uterino es más frecuente que el del cuello, pero menos que el epitelioma. (1)

\section{D - FACTORES PREDISPONENTES}

La frecuencia de diversos factores que aparecen en muchos casos asociados al adenocarcinoma uterino, ha suscitado la posi-

* Del Departamento de Obstetricia y Ginecología del Hospital de San Juan de Dios 
bilidad (1-3-8) de su capacidad predisponente. Tales factores serían los siguientes:

\section{$1-E d a d$}

La edad encontrada en las pacientes afectadas de adenocarcinoma osciló entre los 30 y los 70 años. La literatura revisada parece insinuar que cualquier edad, a partir de la menarquia, es buena para el adenocarcinoma. Sinembargo no encontramos, ni en los archivos consultados ni en los artículos leídos, ningún caso descubierto en el premenarquia.

\section{2 - Miomatosis uterina}

Este factor se encontró en el $25 \%$ de los casos, porcentaje elevado si se tiene en cuenta el escaso número de historias clínicas. Autores, como Masson, revelan un porcentaje del $36 \%$ de adenocarcinomas uterinos sobre 590 pacientes estudiadas por el mismo autor.

A propósito de miomatosis uterina en relación con el adenocarcinoma de endometrio, se cita el caso de una paciente, nulípara, de 49 años de edad, con historia clínica sumamente incompleta y en quien se hace diagnóstico de miomatosis uterina. Intervenida con histerectomía subtotal en el Instituto Materno Infantil, la pieza quirúrgica reveló adenocarcinoma del endometrio con invasión a cuello, a oviductos y a ovarios. En el servicio de Ginecología es reintervenida posteriormente por adenocarcinoma en cuello restante. La evolución post-operatoria, de siete días, se ve empañada por las siguientes complicaciones: deshiscencia de sutura, hidronefrosis bilateral, fístula vésico-vaginal. La última de estas complicaciones aparece como curada en el Archivo del Instituto de Cancerología.

Aparece intervenida por tercera vez, a los dos años, con eventrorrafia. Es de advertir que transcurridos 41 días, a partir de la resección del cuello, acompañada de resección de la cúpula vaginal y de linfadenectomía de la cadena ganglionar ilíaca interna, la paciente fue sometida a tratamiento radioterápico durante 38 días. La evolución post-irradiativa se resume así: parametrios endurecidos en todos los controles; crisis diarreicas atribuíbles a las irradiaciones. Se considera entonces como curada y se cita a examen a los seis meses. Al cabo de cuatro años de la intervención por adenocarcinoma uterino, y abandonado el trata- 
miento, se obtienen en el domicilio de la enferma los siguientes datos: hipertensión arterial, cuadro flebítico que lleva a la paciente al fallecimiento súbito, precedido al parecer de hemianopsia, hemiplejía e intensa disnea.

\section{$3-$ Obesidad}

En la literatura ha quedado perfectamente establecido que la incidencia del adenocarcinoma del endometrio tiene relación indudable con la obesidad. Hoffman (8-15) pudo observar que las dietas con mayor poder calórico aumentan elocuentemente la susceptibilidad a la aparición de este tumor. Ducckklin (8-15) pudo comprobar la relación entre aumento de peso y carcinoma de endometrio. Moss y Novak la señalan como factor predisponente.

En las historias revisadas se encuentran dos casos de los cuales, en uno se hace referencia a la gran dificultad del examen por la severa adiposidad de la paciente y en otro, a las posibles lesiones intensas de las irradiaciones por la misma causa. Una tercera paciente, vista a domicilio, es clínicamente obesa.

No hacemos comentarios especiales sobre la existencia de este factor en relación con situación económica, raza, edad, etc., por no tener informaciones concretas al respecto.

\section{4 - Diabetes}

$\mathrm{Ni}$ los exámenes clínicos ni los de laboratorio revelaron la presencia de esta entidad. Se habla, empero, de la coexistencia del adenocarcinoma del endometrio con la obesidad, síndrome de este último muy ligado a la diabetes.

\section{5 - Hipertensión}

Este signo se encontró en el $54.54 \%$ de los casos, en pacientes con edades entre 46 y 70 años, signos que unidos a la diabetes y a la obesidad, lo consideran algunos autores como sugestivo de un trastorno metabólico, acaso predisponente del adenocarcinoma. Se anota, al respecto, que en los casos aquí estudiados figuran como hipertensas dos pacientes obesas, una colecistectomizada, una tiroidectomizada; otra con hipertrofia nodular del tiroides; la sexta sin antecedentes importantes, fuera de su nuliparidad. 


\section{6 - Hiperplasia endometrial}

La importancia de la hiperplasia como factor predisponente aparece consignada en algunos textos y revistas (1-8-15). El protocolo anatomopatológico no menciona exactamente esta lesión. Se hace referencia a atrofia endometrial, endometrio exuberante, endometrio hemorrágico, endometrio necrótico. "La época de incidencia máxima de la hiperplasia se encuentra en los años premenopáusicos, cuando todavía el cáncer endometrial es bastante raro". No se encuentra relación de las dos entidades durante la vida sexual activa. Durante la menopausia y después de ella, se considera inquietante". Sinembargo, hay casos de tipo dudoso en los cuales ningún Anatomopatólogo puede estar seguro de la naturaleza de la lesión, la cual pasa a ser tratada entonces como cancerosa". (1)

Se ha mencionado la relación entre tumores funcionantes del ovario y exagerada administración de estrógenos, con el adenocarcinoma del endometrio. En cuanto a administración hormonal, solo aparece una paciente con tratamiento estrógeno-progesterónico durante un mes aproximadamente, previo al diagnóstico de malignidad. En cuanto a producción exagerada hormonal, mencionaremos un caso, no incluído en este estudio desde el punto de vista estadístico, de una paciente de 40 años, grávida 9 para 9, con antecedentes ginecológicos normales, que ingresa al servicio de Ginecología con ocho meses de amenorrea; en los dos últimos meses ciclos normales; antecedentes de insuficiencia cardíaca. Se encuentra al examen ginecológico tumefacción hipogástrica que abarca el Douglas, dura, irregular, fija, no dolorosa, deforma el recto plegándolo al sacro; parametrios invadidos; histerometría: $9 \mathrm{cms}$. y discreta hemorragia al examen pélvico; hay ascitis. Se hace diagnóstico de C. A. de Ovario y se toma biopsia endometrial que informa: adenocarcinoma de endometrio. Exámenes de laboratorio previos revelan: anemia, sedimentación acelerada, linfocitosis (este dato de laboratorio es bastante frecuente, debido acaso a la reacción plástica linfocitaria ante el proceso destructivo) ; líquido ascítico hemorrágico, citrino; Rivalta tres cruces; proteínas 8 gramos y negativo para bacilo de Koch; positivo para células tumorales. Sin estudio previo urológico ni de vías digestivas, se considera el caso como inoperable y se remite al Instituto de Cancerología en donde no se hace tratamiento alguno. 
William y Masson Andrews (8) escriben sobre el síndrome de Stein Leventhal asociado con adenocarcinoma del endometrio. Después de hacer algunas consideraciones sobre la posible relación de la hiperplasia endometrial, del tumor de células de la granulosa, de los ciclos anovulatorios, de la infertilidad, de la obesidad, con el adenocarcinoma del endometrio, afirman que la entidad en la cual la estimulación estrogénica prolongada y el adenocarcinoma se hacen presentes, es el síndrome de Stein Leventhal. Se encuentra en estos casos un aumento de la hormona luteinizante, fenómeno semejante al relatado por Sommer y Shermann quienes en un total de 31 casos de adenocarcinoma del endometrio refieren la presencia de hipertrofia pituitaria e hipertrofia adrenal asociadas, asímismo, a un incremento de aquella hormona. Los autores, revisando la literatura, informan sobre 29 casos de conjunción del síndrome con la entidad que nos ocupa, conjunción que acontece en mujeres jóvenes. La resistencia al tratamiento progesterónico, en el sentido de la persistencia de la hemorragia, lleva a los autores a practicar el raspado terapéutico que revela el adenocarcinoma.

\section{7 - Antecedentes obstétricos}

Estos antecedentes se encuentran en mayor relación con el carcinoma del cuello uterino. "El 50\% de las mujeres con carcinoma del endometrio no han tenido familia". La nuliparidad es un factor predisponente muy sugestivo. (1) Se encontró un $58.33 \%$ de pacientes nulíparas en la presente revisión. En dos casos se comprobó la nuliparidad. En relación con la nuliparidad hay quienes piensan sobre la existencia de focos de endometrio inmaduro, indiferenciado, resistentes a la progesterona y sometidos incesantemente a la sola acción estrogénica, la cual llevaría a la menopausia tardía y hemorrágica, con menstruaciones prolongadas, lo cual abonaría el terreno para el nacimiento de la neoplasia.

\section{8 - Adenomiosis}

En las historias revisadas la Anatomía Patológica no revela la presencia de adenomiosis en relación con el adenocarcinoma del endometrio. No se ha podido establecer claramente tal relación, aunque se han registrado concomitancias. (9) 


\section{RESUMEN DE LOS FACTORES PREDISPONENTES DEL ADENOCARCINOMA}

Varios autores comparten la teoría de que todos los factores mencionados obran conjuntamente, en unos casos por congestión y en otros por obstrucción. El último mecanismo, la obstrucción, se explicaría por la defectuosa canalización del material acumulado en la cavidad uterina, material que irritando con su presencia el endometrio provocaría su transformación maligna. (15) La defectuosa canalización estaría dada, para algunos autores, por el estado mismo menopáusico que conlleva atrofia, en ocasiones una verdadera atresia del canal cervical; por la nuliparidad, debido en ella a la estrechez del mismo canal. En nueve de las historias clínicas consultadas, se describen los siguientes hallazgos clínicos y anatomopatológicos: genitales externos, ovarios y oviductos atróficos en 6 casos; cuellos con cervicitis crónica en dos; proliferación anormal de la mucosa endocervical en dos casos; cuello de nulípara en 6 casos; en dos casos abundantes regiones de aspecto poliposo en endocérvix.

\section{$\mathrm{E}-$ HISTOGENESIS}

En el período de formación del tracto genital femenino, derivado de los canales de Muller, el epitelio glandular cilíndrico ciliado que desciende y el epidermoide que asciende, establecen una lucha histológica constante, un desequilibrio inestable, lucha y desequilibrio que se definen en los límites correspondientes al orificio externo del cuello uterino. Cuando la lucha y el desequilibrio persisten, aparecen como mediadores la erosión congénita, la erosión de tipo inflamatorio, los quistes de Naboth, el carcinoma epidermoide del endometrio, el adenoacantoma. Al revisar el Archivo de Anatomía Patológica, se comprobó que en todos los casos, excepto en uno en que no se encontró lesión alguna, el endocérvix permaneció indemne. Sinembargo es de comentar el caso de una paciente de 65 años, no incluída en la serie estudiada, menopáusica desde hacía 25 años y con vida sexual activa. Ingresa al servicio consultando por flujo vaginal fétido desde hace cuatro días, fiebre, escalofríos, dolor en ambas fosas ilíacas y escleróticas ligeramente ictéricas. Al examen ginecológico se encuentra cuello ligeramente rosionado, flujo abundante purulento, fétido; no hay hemorragia. Cuerpo de tamaño, al parecer, normal; fondo de saco derecho empastado y doloroso. Con 
diagnóstico de anexitis aguda y de piometra es sometida a estudio para descartar C. A. de endometrio. Durante su estada hospitalaria el examen bacteriológico del pus arroja una flora Gram positiva. Con tratamiento médico el drenaje cede discretamente, razón por la cual se practica, bajo anestesia, dilatación cervical seguida de la recolección del material extraído. Examinado este material informan carcinoma escamocelular del cuello uterino, grado III. Siete días después una biopsia de endometrio confirma el diagnóstico y la paciente es remitida al Instituto de Carcinología para tratamiento radioterápico.

Nejdat Mulla (7) escribe sobre tres observaciones de adenocarcinoma de endometrio en combinación con adenocarcinoma del cuello uterino. Aunque este autor afirma que no hay información sobre la existencia del adenocarcinoma del endometrio con carcinoma in situ del cuello, trae el relato de un caso comprobado por él. Así mismo informa sobre la presencia de 12 casos de adenocarcinoma endometrial e invasión a cuello. En los casos revisados en el presente estudio la invasión a endocérvix se operó en el $81.88 \%$.

La posibilidad, dice el autor, de la invasión directa o por metástasis desde otro tumor debe ser eliminada, antes de diagnosticar dos neoplasias como tumores separados primarios en el mismo órgano. En este caso cada tumor debe poseer caracteres morfológicos distintos, originarse en sitios diferentes y producirse independientemente.

\section{F - ANATOMIA PATOLOGICA}

Desde el punto devista macroscópico es unánime la aceptación de dos modalidades del adenocarcinoma del endometrio: la circunscrita y ia difusa, términos que describen la impresión que obtiene el examinador ante la visión de la pieza quirúrgica. El término circunscrita conlleva error porque puede haber con anticipación infiltración miometrial.

En las historias revisadas, la lesión se encuentra descrita casi siempre en la siguiente forma: cuerpo uterino aumentado de tamaño (todos los casos); tumor de aspecto papilar (todos los casos, excepto en uno en que el proceso era difuso); infiltración del miometrio (todos los casos); nódulos por debajo del serosa (un caso); invasión anexial masiva (un caso); leiomiomatosis 
concomitante ( 3 casos). No se encontró lesión alguna en un caso correspondiente a una paciente de 35 años quien desde la edad de 33 años presentaba menometrorragias y hemorragia genital continua desde hacía dos meses; antecedentes ginecológicos normales; para 5 con último parto hacía cinco años. Al examen ginecológico se encontró matriz aumentada de tamaño y microadenopatías inguinales duras no adherentes. Sin consignar los caracteres del cuello uterino, el examinador hace el diagnóstico de cervicitis crónica y de ciclo monofásico premenopáusico. Tres días después se practica raspado fraccionado, encontrándose un adenocarcinoma del endometrio en el material obtenido. Se procede a practicar histerectomía abdominal. Sobre la pieza quirúrgica informan: es posible que en el raspado efectuado para tomar biopsia, la lesión haya sido resecada, quedando solo un pedículo no invadido.

La enferma no volvió a examen periódico ni fue posible averiguar sobre su estado actual.

Desde el punto de vista microscópico la lesión usualmente se describe así: proliferación desordenada de glándulas definitivamente neoplásicas; células anaplásicas dispuestas sin arquitectura definida, rodeadas de polimorfonucleares neuirófilos; infiltración profunda del miometrio e invasión del endocérvix; glándulas endocervicales con dilatación considerable y en el estroma infiltración inflamatoria de tipo crónico. Anexos indemnes.

Recordaremos, de paso, la clasificación de Broders (1) basada en la indiferenciación celular: $25 \%$ o menos de células indiferenciadas: grado I. $25-50 \%$ : grado II. $50-75 \%$ : grado III. $75-$ $100 \%$ : grado IV.

En algunos tratados se habla de Adenoma Maligno que correspondería al grado I de la clasificación de Broders; asímismo se menciona el adenoma papilar maligno. Muchos autores están de acuerdo en la abolición de estos términos, adoptando solo el de adenocarcinoma con las dos variedades ya conocidas (circunscrita y difusa).

Pero si bien la clasificación anatomopatológica tiene aceptación unánime, no sucede lo mismo con la clasificación clínica, sobre la cual no existe acuerdo definitivo. La que goza, al parecer, de mayor aceptación es la clasificación de Heymann que conside- 
ra al adenocarcinoma del endometrio como clínicamente operable, técnicamente operable e inoperable. Para otros autores, que miden en cms, el concepto de malignidad, la histerometría es la base de su clasificación: 1 Utero normal con $7,5 \mathrm{cms}$. o menos. 2 Utero de $2 \frac{1}{2}$ meses de embarazo con cavidad menor de $11 \mathrm{cms}$. 3 Utero de 3 meses de embarazo con cavidad de $12,5 \mathrm{cms}$.

En los casos de metástasis ganglionares se lee el siguiente informe anatomopatológico: tumor maligno compuesto por células grandes, poliédricas, con núcleos gigantes y numerosas mitosis. Marcada infiltración secundaria con necrosis hemorrágica. Las células tumorales no adoptan arquitectura alguna que permita diagnosticar su origen.

En cuanto al adenoacantoma, se considera simplemente como un adenocarcinoma en el cual focos ectópicos pre-existentes han sufrido un proceso de metaplasia.

\section{$\mathrm{G}$ - SINTOMAS, SIGNOS, ANTECEDENTES}

En el adenocarcinoma del endometrio encontramos: metrorragia en el $100 \%$ de los casos. Flujo crónico serosanguinolento fétido en el $83.33 \%$. Este síntoma, que frecuentemente pasa inadvertido, tiene en ocasiones un valor diagnóstico precoz, según Stoeckel, por el prurito que ocasiona. El dolor, que siempre revela el compromiso más allá de los límites del órgano, se encontró en un caso de carcinoma de ovario inoperable, propagado a endometrio, a vejiga y a recto.

Como signos clínicos concomitantes: hipertensión en el $54.54 \%$; edema de miembros inferiores en el $100 \%$. Aumento del cuerpo uterino en el $\mathbf{9 9} \%$. Lesiones del cuello uterino en el $\mathbf{2 5 \%}$ en forma de tumefacción que protruye a través del mismo, de proliferación que no permite el paso del histerómetro, de vegetaciones endocervicales múltiples. Hallazgos de formaciones quísticas ováricas en el $16.66 \%$.

\section{$\mathrm{H}$ - COMPLICACIONES, EVOLUCION, CAUSAS DE MUERTE}

Las complicaciones post-operatorias en el adenocarcinoma del endometrio fueron las siguientes: dehiscencia de sutura $(8.33 \%)$; absceso de pared abdominal (16.66\%); eventración $(8.33 \%)$; tromboflebitis $(8.33 \%)$; hidronefrosis bilateral y fís- 
tula vésico-vaginal: un caso. La evolución post-operatoria inmediata se supeditó a las complicaciones antes citadas. La evolución mediata o tardía en tres pacientes vivas ha sido la siguiente: en la primera, dos años siete meses después de la intervención: buen estado general, flujo crónico resistente a todo tratamiento, malestar general, hemorragias profusas, pérdida de peso. En la segunda: edema palpebral bilateral y de ambos miembros inferiores, hipertensión arterial insidiosa año y medio después de la intervención. En la tercera paciente, controlada a los tres años de intervenida, está siendo tratada en Consulta Externa por una Hipertensión arterial irreversible.

Como causas de muerte por adenocarcinoma del endometrio se encontró, en una paciente, un cuadro constituído por flebitis, hipertensión, hemiapnosia, hemiplejía y disnea intensa que llevó al fallecimiento súbito.

Digamos de una vez que el adenocarcinoma es un tumor que parece tener la tendencia a propagarse por extensión directa hacia cuello uterino, anexos y a romper la barrera fisiológica representada por el tejido que le dio origen; solo en tres casos se encontró compromiso linfático regionai; no fue posible comprobar metástasis a distancia.

\section{I - ACIERTOS Y DESACIERTOS CLINICOS}

En el $50 \%$ de los casos de Adenocarcinoma uterino se hizo diagnóstico clínico, corroborado luego por la Anatomía Patológica pre y post-operatoria. En el resto de los casos surgieron como diagnósticos las siguientes entidades: pólipo endocervical; cervicitis crónica-ciclo monofásico premenopáusico. En cuatro pacientes no se hizo diagnóstico.

\section{$\mathrm{J}$ - DIAGNOSTICO DIFERENCIAL}

Los casos de adenocarcinoma del endometrio diagnosticados clínicamente se conșignan de hecho como tales, acaso por la razón aducida de que las hemorragias post-menopáusicas, que no son patognomónicas de cáncer, casi indican su existencia, o de que la metropatía remorrágica, entidad que se menciona como posible en un caso, si bien es una fase frecuente en las postrimerías de la vida sexual, es asímismo el síntoma patológico inicial del adenocarcinoma. 
Entidades como el aborto en sus diferentes modalidades, el parto, la retención de fragmentos, el embarazo extrauterino, la leiomiomatosis, la hemorragia uterina funcional, la colpitis, los pólipos, el carcinoma del cuello, se deben considerar como causas de leucorrea sanguinolenta y de hemorragia en las mujeres premenopáusicas. En las francamente menopáusicas con cuello uterino sano, recordar la poliposis endouterina, los tumores ováricos funcionantes, enfermedades generales hemorragíparas, miomas submucosos, endometritis, endocervicitis y vaginitis seniles.

\section{$\mathrm{K}$ - METODOS DIAGNOSTICOS}

La aspiración endouterina, el escobillonaje endocavitario, son procedimientos que cuentan con adeptos y que tratan de perfeccionarse cada día. La Citología vaginal, también en vía de perfeccionarse y de simplificarse, tiene partidarios que vislumbran la posibilidad de convertirla en un arma eficaz para el diagnóstico y para la prevención. Adolece de una falla: puede suceder que en algunos frotis se encuentren células y en otros no, lo cual acarrearía una falsa impresión de negatividad.

El raspado diagnóstico y la cantidad de tejido extraído (recordar la endometritis senil) son de vital importancia para el diagnóstico. (1) Se menciona el método de Scheffey durante el raspado, que persigue un fin profiláctico y curativo. Efectuada la intervención, el raspado, se hace aplicación intracavitaria de radium y estudio del material obtenido. Si no existe malignidad, se interrumpe dando solo $1.000 \mathrm{mgrs}$. hora con el objeto de promover atrofia endometrial. En caso contrario se prolonga el tratamiento durante las horas que sean necesarias para completar de 4.000 a 8.000 mgrs. Mejorado el estado general, contenida la hemorragia, se procede a intervenir según la técnica elegida.

En los casos aquí revisados se hizo diagnóstico clínico seguido del raspado uterino diagnóstico.

$$
\mathrm{L}-\text { CONDUCTA Y TRATAMIENTO }
$$

En los casos de adenocarcinoma uterino se practicaron las siguientes intervenciones:

9 pacientes - Histerectomía total con salpingooforectomía bilateral. 
1 paciente - Histerectomía subtotal con salpingooforectomía bilateral.

El tratamiento está dado por las conductas seguidas además de la radioterapia post-operatoria a la cual fueron sometidas tres pacientes con el siguiente resultado: sobre la primera paciente aparece la siguiente nota del Instituto de Cancerología: la enfermedad evoluciona desde hace seis meses antes de la intervención con flujo y sin hemorragia franca. Creemos que teniendo en cuenta el resultado histológico (adenocarcinoma del endometrio) y el tiempo transcurrido desde la operación (18 días) no se justifica tratamiento alguno con irradiaciones. Evoluciona luego con hemorragia procedente de cúpula vaginal. Examen en un año. Esto ocurrió el 26 de Noviembre de 1958, fecha en que la paciente se pierde de vista y no es posible conseguirla a domicilio.

En una segunda paciente ni clínica ni anatomopatológicamente pudo hacerse inicialmente el diagnóstico. Las dos entidades consideradas fueron el adenocarcinoma del endometrio y el leiomiosarcoma (a título simplemente informativo consignaremos que el personal científico del I.M.I., en el año de 1960, encontró un rabdomiosarcoma comprobado por la Anatomía Patológica; con este caso ascienden a 15 las neoplasias de este tipo relatadas en la literatura mundial). Pues bien, siguiendo con el caso anterior, la revisión cuidadosa de la biopsia tomada y de la pieza quirúrgica extirpada, reveló un adenocarcinoma del endometrio. Remitida la paciente al Instituto de Cancerología, no fue sometida a tratamiento alguno post-operatorio.

A Radioterapia externa fueron sometidas tres pacientes, una de las cuales vive dos años siete meses después de la intervención y haciendo caso omiso de los exámenes ordenados. Otra muere cuatro años después de la intervención. En una tercera no fue posible conseguir información acerca de su estado actual. A una cuarta paciente, viva dos años después de haber sido intervenida, se le firmó la salida sin orden de exámenes posteriores ni de remisión al Instituto de Cancerología.

En resumen el tratamiento para el Adenocarcinoma del endometrio consistió en la histerectomía total con S. O. B., a excepción de un caso, y complementada con radioterapia post-operatoria. 
"Parece que no hay nadie contrario a la opinión de que la histerectomía total constituye la parte más importante en el tratamiento del Adenocarcinoma uterino. Los mejores resultados se obtienen mediante la aplicación de Histerectomía total con S. O. B., precedidas de la radioterapia. Tiene una desventaja: el intervalo de seis a ocho semanas entre radioterapia e intervención.

\section{CORIOCARCINOMA}

\section{A - DEFINICION}

La definición de Coriocarcinoma obliga a hacer fragmentario el título del presente trabajo, pues se considera, no como un tumor del útero, sino en el útero, ya que derivado del tejido ectodérmico embrionario o trofoblástico, es una neoformación de un individuo que crece e invade los tejidos de otro individuo. Cabría añadir la rara localización del coriocarcinoma en la trompa y en el ovario.

\section{$\mathrm{B}-$ FRECUENCIA}

Se hace hincapié en la pobre frecuencia del coriocarcinoma y quienes presentan estadísticas, se manifiestan renuentes a creer sobre la veracidad de muchos de los casos referidos.

\section{C - FACTORES PREDISPONENTES}

Edad. - La edad, en los cinco casos de coriocarcinoma, osciló entre los 18 y los 42 años, pacientes todas en plena capacidad concepcional. El resto de factores predisponentes, si se descuentan los obstétricos, no parecen tener relación alguna con la aparición de la neoplasia y solo son coincidenciales. "El embarazo, en cambio, aunque algunos autores consideren lo contrario, es condición indispensable para que se produzca el coriocarcinoma". En dos casos de mola vesicular, Bleier (4) pudo comprobar una toxoplasmosis latente. En una de estas mujeres, cuatro embarazos de dos diferentes maridos originaron cada vez una mola vesicular. De Ruyck (4) obtuvo un virus coriotropo de una mola vesiculosa, el cual reinoculado a animales de experimentación originaron molas vesiculosas. "El $50 \%$ de molas vesiculosas degeneran en coriocarcinoma”.

Y al hablar de posible degeneración sería oportuno recordar, desde el punto de vista de la patogenia de esta lesión, que el epi- 
telio corial del feto de desarrollo normal y con vellosidades normales, tiene la propiedad de penetrar en la decidua para infiltrarla. Tal capacidad proliferante y proteolítica viene frenada por la función defensiva del tejido materno; biológicamente en la decidua se forma el fermento antitripsina contra la tripsina de la célula del epitelio corial. El defectuoso desarrollo del feto o su expulsión traen a veces como consecuencia la falta de esta función de freno contra el epitelio corial; lo inmediato es que las células de este epitelio aumenten su capacidad proliferante y proteolítica con el resultado de una mola o de un coriocarcinoma.

\section{D - HISTOGENESIS}

El Coriocarcinoma deriva del trofoblasto, epitelio de aquella envoltura embrionaria que se pone en comunicación con los tejidos maternos, es decir con el útero y posibilita de este modo la alimentación del embrión. La capa celular primitiva se denomina citotrofoblasto que luego se convierte en sincitio, llamado sincitio trofoblasto o espongiotrofoblasto. No mencionaremos en este trabajo lo referente al coriocarcinoma testicular.

\section{$\mathrm{E}-$ ANATOMIA PATOLOGICA}

Desde el punto de vista macroscópico el coriocarcinoma se describe, en textos y revistas de la siguiente manera: masa grumosa, obscura, ulcerada, hemorrágica que invade el tejido muscular. La información que nos da la Anatomía Patológica en los casos revisados aquí se resumen así: cuerpo uterino aumentado de tamaño; presencia de cuerpos amarillos numerosos; hallazgo de vesículas en algunos casos; tejido friable, esponjoso, hemorrágico e infiltración del miometrio.

Al microscopio se encuentran células trofoblásticas de apariencia tumoral, ausencia de vellosidades coriales y quistes ováricos múltiples. Lo que más llama la atención es la ausencia de vellosidades coriales, la disposición alveolar del trofoblasto que invade músculo y las áreas de necrosis y de hemorragia.

\section{F - SINTOMAS Y SIGNOS}

En el coriocarcinoma encontramos metrorragia en el $100 \%$ de las pacientes. Dolor de tipo expulsivo en íntima relación con la metrorragia en todos los casos. Ictericia en el $41.66 \%$. Hemoptisis en un caso. 
El diagnóstico del coriocarcinoma se hará en forma casi certera a base de los antecedentes (aborto, parto, mola hidatidiforme, hemorragia que no cede con el raspado ni con las medidas hemostáticas habituales) ; una historia clínica completa, la valoración del estado general, la dosificación gonadotrópica y el examen anatomopatológico, todo considerado en conjunto. Pero aun así el diagnóstico resulta en ocasiones difícil, como ocurrió con una paciente de 42 años, con último parto hacía tres años y que ingresó al servicio de Ginecología (Consulta Externa) por amenorrea de 7 meses y con hemorragia vaginal continua subsiguiente, sensación de tumefacción abdominal y dolor de tipo expulsivo en el bajo vientre; intervenida con raspado, cesa la hemorragia que reaparece luego en forma de flujo serosanguinolento acompañado de dolor difuso abdominal, malestar general, signo de Murphy francamente positivo, constipación y microadenopatías inguinales. Un examen practicado en Consulta Externa por el personal más capacitado de Ginecología consigna los siguientes hallazgos clínicos: "tumefacción abdominal de pubis a ombligo, renitente, dolorosa, poco móvil, no adherida a la piel. Conclusión: útero de 4 veces el tamaño normal, doloroso a la palpación y espontáneamente; flujo piosanguinolento. Histerometría: 13 cms.". Se consideran entonces las siguientes posibilidades diagnósticas: coriocarcinoma, embarazo, hiperreactio luteinalis infectados, adenocarcinoma del endometrio, endometritis, anexitis. La evolución, casi inmediata una vez hospitalizada la paciente, consistió en fiebre, algia de miembro inferior izquierdo, dolor hipogástrico, vómito, ictericia. Un Galli cuantitativo ascendió a 500.000 unidades. Se duda del coriocarcinoma y la posibilidad del embarazo subsiste. Se toma biopsia de endometrio que revela endometritis. Una radiografía simple de abdomen es negativa para embarazo. Decrece la tumefacción y se practica una nueva histerometría de 9,5 cms. Surge la sospecha de una mola vesicular; el Galli se mantiene, al segundo examen, en 500.000 unidades. La dosificación biológica obliga a la intervención consistente en histerectomía total con anexectomía bilateral. Luego de nueve días de post-operatorio, y con una estada hospitalaria de 45 días, la paciente fallece. El protocolo anatomopatológico reveló una mola vesicular degenerada en coriocarcinoma con invasión venosa. La necropsia informa: ascitis e hidropericardio. Mujer de 40 años que muere con corioadenoma de struens, por peri- 
tonitis aguda, bronconeumonía terminal y nefrosis hemoglobinúrica.

La relativa benignidad o la franca malignidad del corioadenoma antes citado se encuentran en discusión.

El promedio temporal de la aparición sintomática y signológica del coriocarcinoma oscila entre meses y años, al parecer. De tres pacientes analizadas en esta serie, en la primera el último parto había tenido lugar hacía tres años; en la segunda un aborto había ocurrido hacía dos años; en la tercera figura la siguiente: paciente de 18 años, nulípara y con antecedentes ginecológicos normales. Ingresa al Instituto Materno Infantil en donde se hace diagnóstico de aborto inminente. Se interviene con raspado y se hace diagnóstico operatorio de aborto molar. Noventa días después reingresa la paciente y se encuentra una matriz aumentada de tamaño como para uno y medio meses de embarazo con fondos de saco ocupados por tumefacciones de 5 a $7 \mathrm{cms}$. de diámetro. Se consideran entonces dos posibilidades diagnósticas: 1mola vesiculosa. 2-Coriocarcinoma. Se practica nuevo raspado que lleva al siguiente informe anatomopatológico: tumor de $5 \mathrm{cms}$. esponjoso, hemorrágico, infiltración al miometrio y al endocérvix; no hay vellosidades coriales. Simultáneamente con el diagnóstico clínico y el raspado diagnóstico, se ordenan exámenes biológicos en número de tres: los dos primeros, con treinta días de intervalo, resultan negativos. El tercero, positivo al mes y con la paciente en control ambulatorio, precisa una investigación cuantitativa que se manifiesta así: $160.000-20.000-80.000-20.000$ con intervalos de 25 días. Recordemos de paso que la negatividad del Galii no excluye la presencia del coriocarcinoma, pues las remisiones en el curso evolutivo así como las necrosis extensas de la neoplasia pueden producirla.

Ante la persistencia del Galli, se solicita interconsulta al Departamento de Ginecología en donde consideran la posibilidad de un Corioadenoma destruens y se aconseja practicar nuevo raspado y estudio radiográfico pulmonar. El personal de Obstetricia, reunido en pleno, decide la histerectomía total con salpingooforectomía bilateral.

No figura en la historia control posterior ni tratamiento alguno subsiguiente. Desafortunadamente no fue posible localizar a la paciente en su domicilio. 


\section{G - EVOLUCION Y CAUSAS DE MUERTE}

De coriocarcinoma fallecieron dos pacientes: la primera, de 42 años, luego de un cuadro septicémico fulminante, muere por peritonitis aguda, bronconeumonía terminal y nefrosis hemoglobinúrica en el tercer día post-operatorio.

La segunda por infarto cardíaco, infarto pulmonar, neumonía izquierda, cor pulmonale agudo, diagnósticos todos realizados en el Servicio de Medicina Interna 21/2 años después de la intervención aproximadamente. No se practicó en aquella ocasión examen alguno de laboratorio ni necropsia.

\section{$\mathrm{H}$ - ACIERTOS Y DESACIERTOS CLINICOS}

En los casos aquí revisados el diagnóstico de coriocarcinoma fue patrimonio del interrogante y la Anatomía patológica solo fue concluyente en dos casos, pues en el resto primó la dosifieación de las gonadotropinas en el diagnóstico y en el tratamiento.

\section{I - DIAGNOSTICO DIFERENCIAL}

En los casos de coriocarcinoma es preciso tener en cuenta todas las causas de hemorragia mencionadas en los casos de adenocarcinoma del endometrio y correspondientes a mujeres en plena capacidad concepcional, además de la mola vesicular. La endome. tritis sincitial y el corio-adenoma destruens precisan siempre la biópsica confirmadora.

\section{J - METODOS DIAGNOSTICOS}

El raspado terapéutico comprobó dos casos; en los restantes el diagnóstico fue dudoso y la conducta seguida se supeditó a la positividad persistente de las pruebas biológicas, positividad que osciló entre 20.000 y 500.000 unidades. Recordaremos en este sentido que el embarazo gemelar, la hiperemesis gravídica elevan la concentración de gonadotropinas.

Pero la negatividad del raspado practicado con fines diagnósticos no prueba la ausencia del coriocarcinoma ya que la neoplasia, empotrada en el músculo uterino, puede escapar fácilmente a la acción de la cureta. Lo mismo diremos de la negatividad del Galli que simplemente puede obedecer a remisiones intermitentes en el curso evolutivo de la lesión o a necrosis extensas 
que impiden una buena comunicación para el paso de las gonadotropinas a la sangre materna.

La dosificación biológica en el líquido cefalorraquídeo, de ningún valor para muchos autores en el sentido de un diagnóstico diferencial con la mola, no se practicó en ningún caso.

En dos pacientes el estudio radiológico orientó el diagnóstico al revelar metástasis de localización pulmonar.

Fuera de este hallazgo, y de los fenómenos ictéricos que podrían suponer un compromiso hepático, no se encontraron proliferaciones ectópicas ni en vagina, ni en cerebro ni en riñones, proliferaciones que para Huber y Horman (4) son benignas y excepcionalmente de naturaleza maligna. De acuerdo con estos autores se designa como corioepiteliosis todas las proliferaciones coriales ectópicas benignas, corioepieteliosis que puede ser interna (mola vesicular, por ejemplo) o externa (proliferaciones benignas que aparecen en vagina, pulmón, cerebro, etc.). "De estas corioepiteliosis se separa el coriocarcinoma puro con su curso morboso característico fulminante. De esta interpretación de los hechos clínicos deriva la consecuencia de que aun en presencia de metástasis coriales, por ejemplo en vagina, no se hace necesaria la extirpación del útero; además en caso de disminución progresiva de la concentración hormonal y respecto a la ejecución de medidas quirúrgicas radicales, en cada caso, y sobre todo si se trata de mujeres jóvenes, se ha de ser muy reservado.

Las formas que retrogradan espontáneamente o con tratamiento médico o con intervenciones incompletas, quizá corresponderían a esta clase de comportamiento corial.

$$
\mathrm{K} \text { - CONDUCTA Y TRATAMIENTO }
$$

En los casos de coriocarcinoma se practicaron las siguientes intervenciones :

Histerectomía total con Salpingooforectomía bilateral en 3 pacientes.

Histerectomía subtotal en una paciente.

Histerectomía "ampliada" en una paciente.

Al Instituto de Cancerología fue enviada una paciente sobre quien aparece el siguiente informe: esta enferma, en vista de sus 
metástasis pulmonares, solo tiene posibilidades de tratamiento a base de adrenelectomía, de mostazas y sus derivados. Por desgracia, dada las circunstancias del Instituto, se le da salida. Se trata de la paciente que falleció $2 \frac{1}{2}$ años después de la intervención en el servicio de medicina interna con un cuadro cardio-pulmonar diagnosticada clínicamente.

En resumen el tratamiento quirúrgico del coriocarcinoma consistió en la Histerectomía total con Salpingooforectomía bilateral, a excepción de un caso en que se practicó histerectomía subtotal. Ninguna paciente fue sometida a radioterapia post-operatoria, pero sí figura el tratamiento médico en un caso con hemoptisis, a base de metrotapsato, mustrón, droga que no fue posible conseguir. En la literatura revisada se encuentran relatados dos casos tratados médicamente. Uno de ellos con etil-uretano por vía intraarterial, vía que se considera como la preferida por la rapidez con que obra sobre la localización primitiva tumoral y sobre las metástasis. El segundo caso aparece tratado con aminopterin, un antifólico. En ambos la supervivencia es de un año hasta el último examen periódico. Se especula sobre la posibilidad del tratamiento médico del coriocarcinoma.

Según Hohlweg y Bauer (4) con dosis diarias de 10 a 100 mgrs. de diacetato de dienoestrol se logra la involución de las metástasis pulmonares. Perrault (4) recomienda Ia P-oxipropiofenona con el mismo fin: el tratamiento consiste en 0.3 gramos diarios por vía oral; en suma, 120 gramos en seis meses.

\section{L - RESUMEN Y CONCLUSIONES}

1 - Se presenta un trabajo de recopilación estadística sobre adenocarcinoma del endometrio y coriocarcinoma en un lapso de siete años comprendido entre los años de 1953-1959.

2 - Se habla sobre factores predisponentes de estas neoplasias.

3 - Se hacen algunas consideraciones sobre histogénesis y patogenia.

4 - Basados en el protocolo de Anatomía Patológica se describen las lesiones desde los puntos de vista macro y microscópico.

5 - Se relatan los síntomas y signos, recurriendo directa- 
mente al cuadro clínico de las enfermas, muchas de las cuales aparecen como ejemplos ilustrativos.

6 - La apreciación, las complicaciones, la evolución y las causas de muerte son el resultado de la revisión de las historias clínicas, de la entrevista personal y del auxilio de la necropsia.

7 - Las conductas seguidas y el tratamiento efectuado se ciñen exactamente al criterio del Servicio de Obstetricia y Ginecología en donde estuvieron hospitalizadas las pacientes, citando a la vez la conducta y el tratamiento de otras escuelas.

\section{CONCLUSIONES}

1 - La corta serie estudiada no permite deducir conclusiones de importancia.

2 - En textos y revistas se habla de factores etiológicos. Aquí nos referimos a factores predisponentes.

3 - La histogénesis del adenocarcinoma permanece sin confirmación definitiva. Es más clara la del coriocarcinoma.

4 - Desde el punto de vista anatomopatológico, primó la forma circunscrita sobre la difusa del adenocarcinoma del endometrio. Da la impresión de que el adenocarcinoma del endome-trio es una lesión relativamente benigna, de buen pronóstico, si se descubre a tiempo. Tal consideración se desprende del aspecto macroscópico de la lesión que trata de circunscribirse a la barrera del tejido que le dio origen y asímismo por el relativo buen estado general de las pacientes.

Anatomopatológicamente el coriocarcinoma aparece como de diagnóstico difícil.

5 - Resaltan como síntomas del adenocarcinoma del endometrio la metrorragia meno y post-menopáusica; la hipertensión, como signo, podría estar ligado a otras entidades de tipo cardiovásculorenal; el aumento del tamaño uterino es casi constante. La nuliparidad, como antecedentes, llama la atención por su elevada frecuencia.

En cuanto al coriocarcinoma los síntomas, los signos, los antecedentes deberán estar estrechamente ligados a la dosificación biológica y al estudio anatomopatológico para interpretarlos en conjunto. 
6 - De los ejemplos ilustrativos (una paciente que se intervino y en la cual no se encontró lesión en la pieza quirúrgica extirpada) surge la duda acerca de si esta lesión se extirpó evidentemente con el raspado o de si hubo error diagnóstico previo a la intervención y se practicó una operación innecesaria.

7 - La extensión por continuidad y la vía linfática aparece en los casos revisados como las preferidas por el adenocarcinoma del endometrio.

El coriocarcinoma se propaga rápidamente por vía sanguínea.

\section{BIBLIOGRAFIA}

1 NOVAK E. Tratado de Ginecología. Editor Fresnada (La Habana, Cuba) 5a Ed. 1955.

2 STOECKEL, W. Tratado de Ginecología. Versión española de la última alemana. Editora Nacional (México) 1952.

3 CURTIS HUFFMANN. Ginecología. 3: Ed. Edit. Salvat (Madrid) 1955.

4 PSCHYREMBEL, W. Obstetricia práctica. Editorial Labor, S. A. Barcelona-Madrid. 1958.

5 FISCHEL, A. Compendio de Embriología Humana. Editorial Labor. 2 Ed. Barcelona, Madrid, 1951.

6 Am. J. Obstetrics and Ginecology.

7 NEJDAT MULLA, M.D. Youngtown, Ohio. Vol. 79 N․ 2 Febrero de 1960.

8 WILLIAMS, MASSON ANDREWS, Norfolk, Virginia. Vol. 80 N: 41960.

9 STEWART MARCUS. Palo Alto, California. Vol. 81 № 21961.

10 Obstetricia y Ginecología Lationamericanas.

11 AHUMADA JUAN CARLOS. Vol. XV Agosto de 1957.

12 WILDER JULIO. Vol. XVI Agosto de 1958.

13 PEPE ANTONIO. GUGLIEME PEDRO. ACOSTA JORGE. Vol. XIII Marzo-Abril 1955.

14 Obstetricia y Ginecología de México.

15 GALLO DELFINO. Vol. XII 1957.

16 INFANTE ANGUSTO, VELASQUEZ TOMAS. Vol. XII 1957.

17 FRAGOSO LIZALDE DAVID. Vol. XV Nং 84 Enero-Febrero de 1960.

18 FRAGOSO DAVID. Vol. XV N: 87. Julio-Agosto de 1960. 